\title{
Addendum: Kienböck Disease: A New Algorithm for the 21st Century
}

\author{
David M. Lichtman, MD ${ }^{1,2}$ William F. Pientka II, MD \\ 1 Department of Surgery, Uniformed Services University, \\ Bethesda, Maryland \\ ${ }^{2}$ Department of Orthopaedic Surgery, University of North Texas \\ Health Science Center, Fort Worth, Texas \\ ${ }^{3}$ Department of Orthopaedic Surgery, John Peter Smith Hospital, \\ Fort Worth, Texas \\ ${ }^{4}$ Department of Orthopaedic Surgery, Flinders University, Adelaide, \\ South Australia, Australia \\ ${ }^{5}$ Flinders Medical Centre, Adelaide, South Australia, Australia
}

J Wrist Surg 2017;6:e1-e2.

\section{Modification of the Lichtman Bain Classification}

After publication of the above article in the Journal of Wrist Surgery, Volume 6, Number 1, 2017 (DOI: 10.1055/s-00361593734), the authors would like to make minor modifications to reflect the importance of the carpal instability of Kienböck's disease, which is then followed by the degenerative changes within the carpus.

In this new classification, the " $\mathrm{C}$ " category highlights the effect of Kienböck's disease on the wrist. The secondary effects on the wrist usually begin with instability of the proximal carpal row, with flexion of the scaphoid (RSA $>60^{\circ}$, Lichtman
Gregory Ian Bain, MBBS, FRACS, FA, PhD ${ }^{4,5}$

Address for correspondence Gregory lan Bain, MBBS, FRACS, FA, PhD, 196 Melbourne Street, North Adelaide, 5006, South Australia, Australia (e-mail: greg@gregbain.com.au).

IIIB). This is followed by the progressive secondary degenerative changes within the carpus. It will usually commence in the radiolunate articulation, and progresses to the radioscaphoid and finally the midcarpal joint. However, there is some variability of the distribution of the degenerative changes (e.g., a coronal lunate fracture can predispose to earlier degeneration in the midcarpal joint). Further research is required to determine the exact details of the degenerative changes in Kienbock's disease.

With advanced degeneration involving all articulations, the only option is a salvage procedure such as a wrist fusion.

The updated algorithm appears as follows. 


\section{A. Patient's age?}

A1. $<15$ years - Non-operative

A2. 16 -20 years - Non-operative first. Consider unloading procedure.

A3. $>70$ years - Non-operative first. Consider synovectomy and / or follow algorithm below.

\section{B. Stage of the lunate?}

\section{B1. Lunate intact - Protect / unload the lunate}

(Cortex and cartilage intact - Lichtman 0, I, II, Schmitt A, Bain 0)

Orthosis or cast first (trial for 2-3 months)

Radial shortening osteotomy, capitate shortening for ulnar $+v e$ (radial epiphysiodesis*)

(Alternatives - Lunate decompression, vascularized bone graft*, radius forage*)

\section{B2. Lunate compromised - Consider lunate reconstruction}

(Localized lunate disease - Lichtman IIIA, Schmitt B, Bain 1)

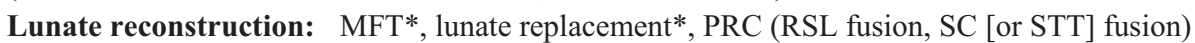

\section{B3. Lunate not reconstructable - Lunate salvage}

(Advanced lunate disease - Lichtman IIIC, Schmitt C, Bain 2b)

Lunate salvage (excision): Lunate replacement*, capitate lengthening, PRC (SC fusion)

\section{State of the wrist?}

C1. Carpal instability with intact articulations - Stabilize

Typical scaphoid flexion, with RSA $>60$ degrees (Lichtman IIIB).

Stabilize radial column (SC fusion)

\section{C2. Localized carpal degeneration - Reconstruct}

C2a. Radiolunate articulation compromised (Lichtman IIIA, Bain 2a)

Bypass (SC fusion), reconstruct (MFT graft) or replace (lunate prosthesis), Fuse (RSL fusion)

\section{C2b. Radioscaphoid articulation compromised}

PRC if lunate facet intact, RSL fusion if lunate facet compromised and midcarpal joint intact.

\section{C3. KDAC, Advanced carpal collapse and degeneration - Salvage}

Wrist not reconstructable (advanced wrist disease - Lichtman IV, Bain 4)

Salvage (fusion or arthroplasty).

Other options that can be considered have been placed in (parentheses). STT fusion is an alternative to SC fusion. *Alternate procedures, techniques that require specialized skills and therefore affect what the surgeon can offer. The classification determines the recommended treatment based on the patient's age (A), status of the lunate (B) and the status of the wrist (C). What the surgeon can offer (D) and what the patient wants (E) ultimately determine what is performed. 